\title{
Fatigue Test under Mode II+III Mixed-Mode Condition.
}

\author{
Masanori Kikuchi \\ Department of Mechanical Engineering, Faculty of Science and Technology, Science University of Tokyo, Noda, \\ Chiba, 278-8510, Japan
}

\begin{abstract}
Almost all of fatigue crack growth in the structure occurs under the mode I loading. However in some structures, the fatigue may occur not only under the pure mode I condition but also in the shear and torsion conditions. Under such conditions, the mixed mode fatigue crack growth may occur. In this paper, mode II + III fatigue test is conducted using a single-axis testing machine. After the test, the fracture surface is observed using SEM and the characteristic feature of the mixed mode fatigue surface is observed. The three-dimensional FEM analysis is carried out and stress intensity factors, KII and KIII, are evaluated. The crack growth direction is compared with that predicted by Erdogan-Sih criterion. The crack growth rate is evaluated and is compared with that of mode I condition.
\end{abstract}

\section{INTRODUCTION}

In the fatigue crack problem, almost all of the fatigue crack occurs under the mode I loading. Then the studies on the mode I fatigue problems have been conducted by many authors and a lot of papers have been published. However, in the real structures, the fatigue crack may occur not only under the pure mode I condition but also in the shear or torsion conditions. Under such conditions, the mixed mode fatigue crack growth may occur. Several papers have been published on the mixed mode fatigue problem. In these studies, the pure mode II or I+II tests are conducted successfully, and some special tools for the mode II test are proposed. But many of them use thin specimen, and the thickness effect is not considered. On the mode III test, a torsion specimen is widely used[1]. To avoid the contact of crack surfaces, the tensile load is given at the same time. For this purpose, two-axis testing machine is needed. In the present study, the mixed II+III mode fatigue test is conducted using a single-axis testing machine. The test specimen is a four-point shear specimen with a side crack. To avoid the fatigue surface contact, the static mode I load is given. The fatigue fracture surface is observed by SEM(Scanning Electron Microscope) and found that the fatigue crack grows in the initial crack plane, which can not be predicted by the Erdogan-Sih criterion[2] considering the out-of-plane shear stress. The three dimensional FEM(Finite Element Method) analysis is conducted. By modeling the fatigue crack growth behavior, the change of the stress intensity factors along the crack front is studied. The crack growth rate is evaluated and is compared with that of pure mode I fatigue, and the results are discussed.

\section{EXPERIMENTS.}

The material used in the experiment is Aluminum alloy A2017-T4. The chemical compositions and the mechanical properties of this material are shown in Table 1 and 2, respectively. The shape and the dimensions of the specimen are shown in Figure 1. At the center of the specimen, a notch is introduced by EDM(Electric Discharge Machining) method, and the fatigue crack by pure mode I loading is introduced. The final crack length is $15 \mathrm{~mm}$, which is a half of the specimen width.

Table 1 chemical composition

\begin{tabular}{|c|c|c|c|c|c|c|}
\hline $\mathrm{Si}$ & $\mathrm{Fe}$ & $\mathrm{Cu}$ & $\mathrm{Mn}$ & $\mathrm{Cr}$ & $\mathrm{Zn}$ & $\mathrm{Mg}$ \\
\hline 0.61 & 0.32 & 4.18 & 0.70 & 0.03 & 0.19 & 0.54 \\
\hline
\end{tabular}

Table 2 mechanical properties

\begin{tabular}{|c|c|c|c|}
\hline$E[\mathrm{GPa}]$ & $\nu$ & $\sigma_{Y}[\mathrm{MPa}]$ & $\sigma_{B}[\mathrm{MPa}]$ \\
\hline 70 & 0.33 & 340 & 460 \\
\hline
\end{tabular}


The four-point shear load is subjected to this specimen. The distributions of KI, KII and KIII along the crack initial front are evaluated using FEM (Finite Element Method) and VCCM (Virtual Crack Closure Method) method[3]. The results are shown in Figure 2. The abscissa of this figure is the specimen thickness, where $2 \mathrm{z} / \mathrm{B}=0$ means the mid-plane of the specimen, and $2 \mathrm{z} / \mathrm{B}=1.0$ and -1.0 means the surface of the specimen. The KI component keeps nearly zero along the crack front. Near the surface, KII becomes very large, but is decreases inside of the specimen. KIII is large in whole location along the crack front. At the mid-plane of the specimen, there exists pure mode III condition. As KI is nearly zero along the crack front, it is estimated that the contact between fatigue crack front may occur after some amount of the crack growth. The contact of fatigue crack surfaces decreases the effective stress intensity factor range, and becomes difficult to neglect the effect of the contact when the crack growth rate is studied. To avoid the fatigue crack surface contact, the static mode I load is given using some special tool as shown in Figure 3. The final loading condition of this specimen is shown in Figure 4. By changing $\delta$ value in the figure, the amount of the mode I load is controlled. The result is shown in Figure 5 , where $\delta$ is determined to be $0.1 \mathrm{~mm}$. The differences between the displacements of both crack surfaces are shown in this figure. The crack length is changed from $a / W=0.5$ to 0.933 . For $a / W=0.5$, the curvature of the crack front is considered. It is the results of the measurement of the real specimen. For larger a/W value specimens, a straight crack front is assumed. For every case, the displacement differences show plus values, which mean that the contact between both surfaces could be avoided. In the fatigue test, $\delta$ is kept to be $0.5 \mathrm{~mm}$.

During the fatigue test, the crack tip behavior is observed by CCD camera and is recorded in the computer. The fatigue crack growth rate at the surface of the specimen is obtained.
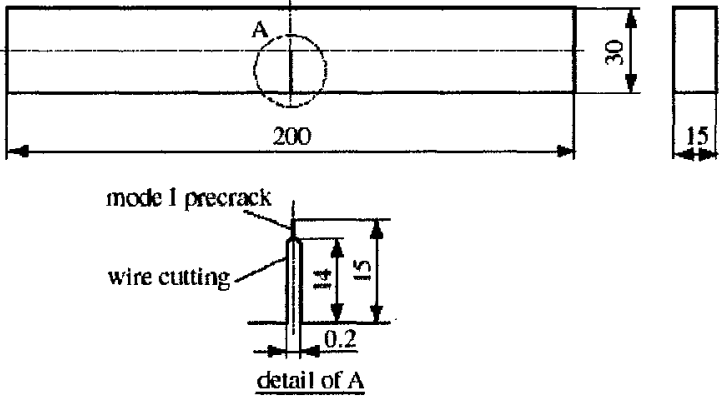

Figure 1 Test specimen.

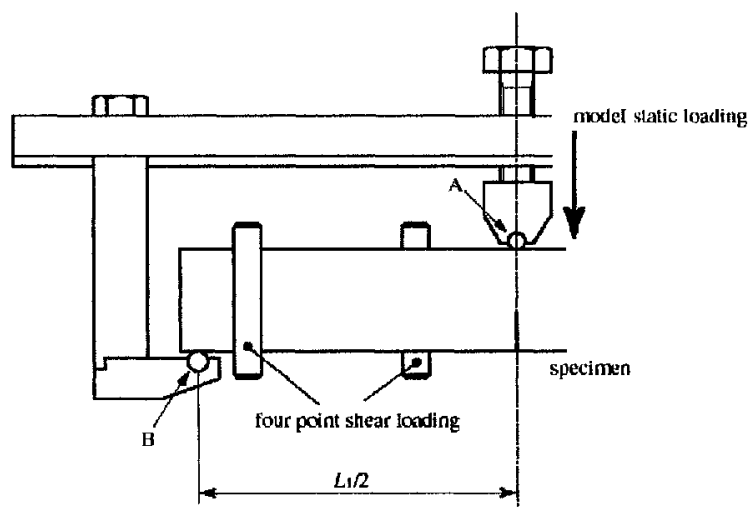

Figure 3 Mode I loading tool.

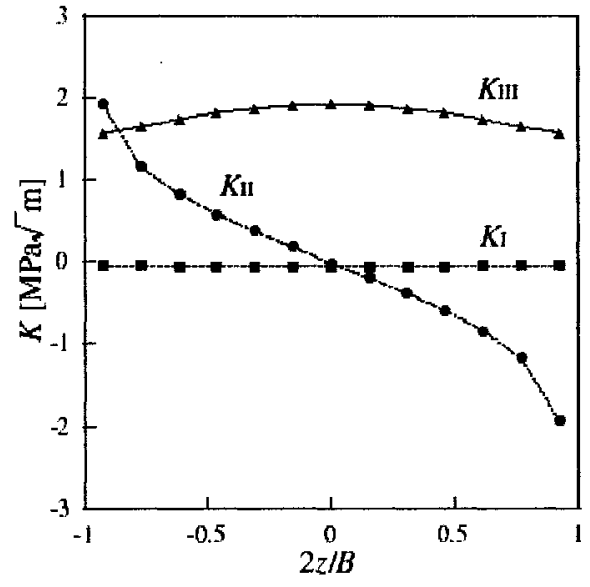

Figure $2 \mathrm{KI}$, KII and KIII distributions.

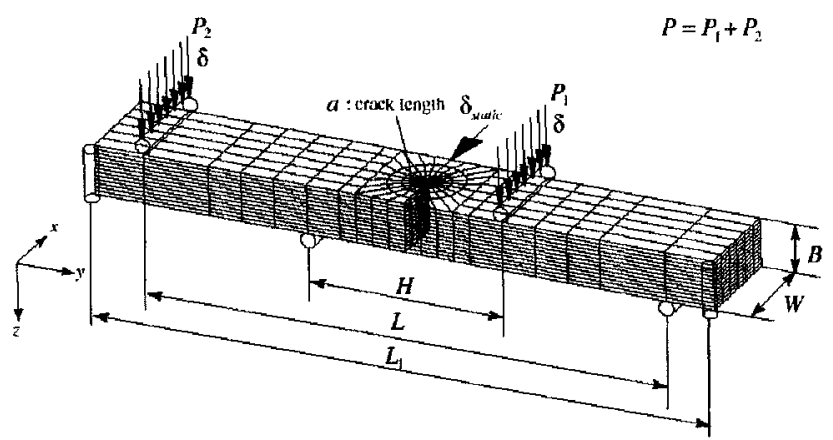

Figure 4 Final loading condition 


\section{FATIGUE CRACK SURFACE OBSERVATION.}

The crack growth direction on the specimen surface is shown in Figure 6. The crack grows along the initial crack tip direction. All specimens showed the same results. The crack surface is shown in Figure 7. In the whole fracture surface, the crack growth direction is same, and is parallel to the initial crack surface. But the fracture surface is not smooth, as is in pure mode I fatigue, and is waving. It is also noticed that the crack growth begins at the specimen surface, and grows to the inside of the specimen. It occurs from both specimen surfaces, and finally two fatigue cracks meet at the mid-plane of the specimen. Before they meet at the mid-plane, the fatigue crack under mixed mode condition intersects with the initial crack front, which is generated by the mode I fatigue. After meeting at the mid-plane, it grows forward together. During these whole processes, the fatigue crack front exists in the same plane as the initial crack surface. In the pure mode I fatigue, the fatigue crack grows faster inside of the specimen than near the specimen surfaces. It is completely different from that of mode II +III mixed mode conditions. At last, some black area appears on the fatigue crack surface. It is made by the contact between crack surfaces. It means that the contact between crack surfaces could be avoided during almost all of fatigue crack growing processes.

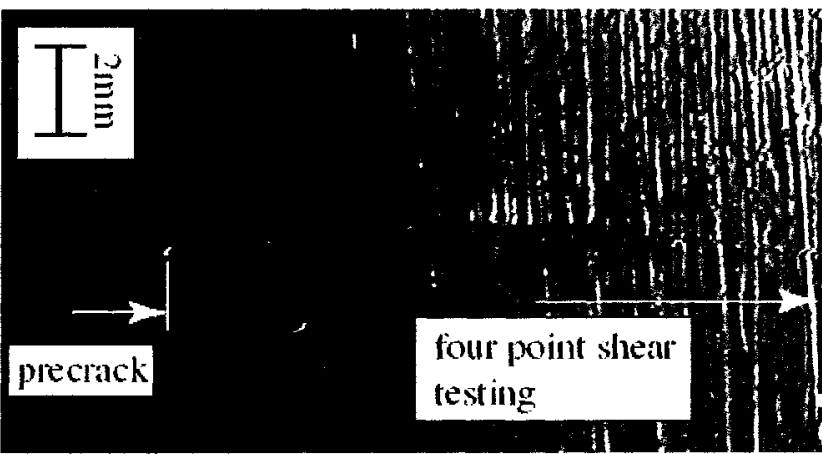

Figure 6 Fatigue crack growth direction.
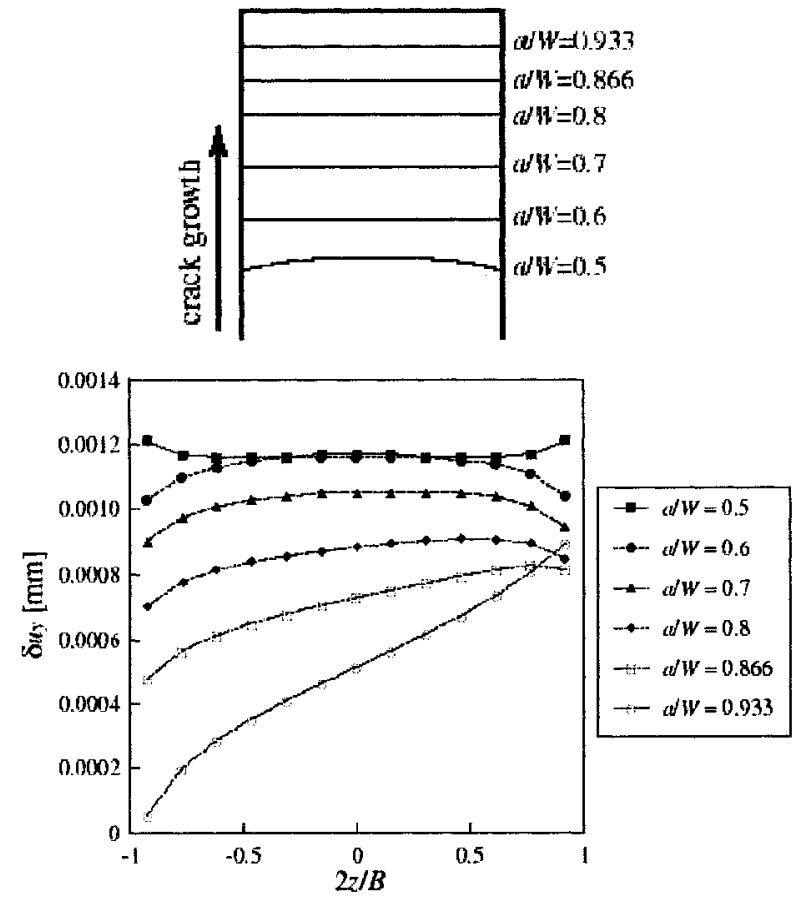

Figure 5 Crack surface opening.

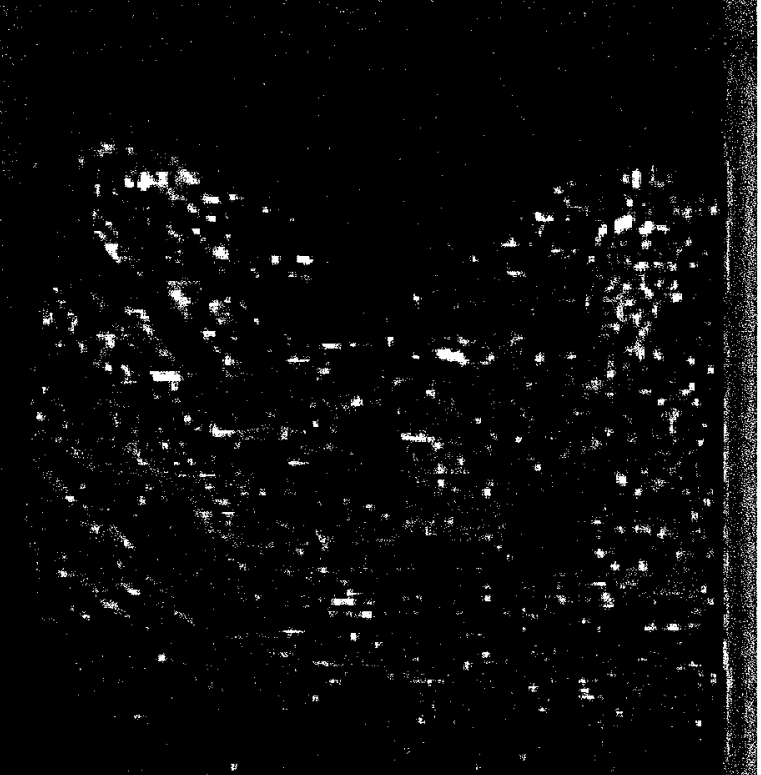

Figure 7 Fatigue crack surface. 
Figure 8 is the SEM photo of the fatigue crack surface. Macroscopic fatigue crack growth direction is from the bottom to the top of this photo. Fatigue surface is made of many laminates. It is similar to the fatigue surface by pure mode II loading shown by Otsuka et al[4,5]. They are neither parallel nor perpendicular to the crack growth direction. It is the combination of in-plane and out-plane shear modes. The width of each laminate is 0.5 to 2 micrometers. It changes with the change of the fatigue crack growth rate. It is estimated that each lamina is generated by one cyclic load. But they are not striation because they are not perpendicular to the crack growth direction.

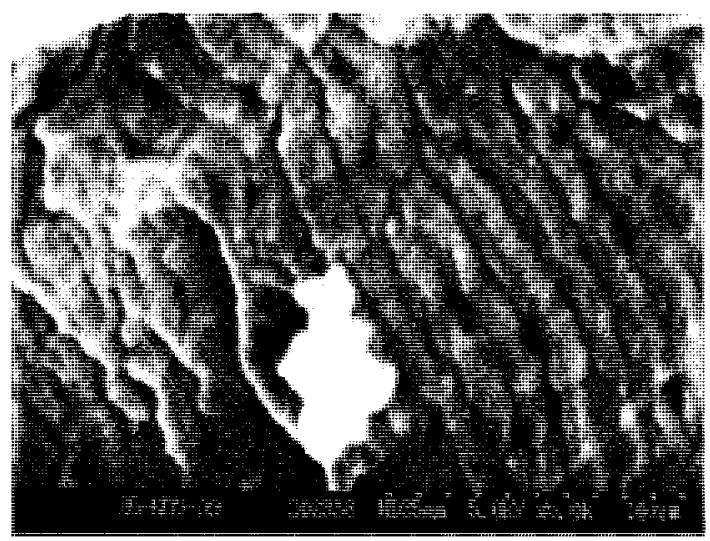

Figure 8 SEM photo of fatigue surface.

\section{EVALUATION OF STRESS INTENSITY FACTORS AT THE CRACK TIP.}

Three-dimensional FEM analyses are conducted, and the stress intensity factors at the crack tip are evaluated. As the crack grows faster near the specimen surface than that of inside, the FEM model is made as shown in Figure 9. They are made based on the experimental observation. In the real specimen, crack front is not straight as in this figure. Then the average configuration is used for the modeling. By changing the shape of the fatigue crack front, the fatigue crack growth is modeled. Each model is called by the value of the crack length with respect to the specimen width, $a / W$, where $a$ is the crack length measured at the specimen surface. $a / W=0.5$ is the initial state, without mixed mode fatigue crack growth. When a/W becomes 0.8 , both fatigue cracks from the specimen surface meet at the mid-plane of the specimen. Along the crack front, stress intensity factors, KI, KII and KIII are evaluated using VCCM method. The energy release rate, GI,GII and GIII are evaluated based on the $x-y-z$ coordinate system in the global field. But by the fatigue crack growth, the crack front configuration changes, and mode II and III changes with the crack growth. As shown in Figure 10, the coordinate system is attached to the crack front, and GII and GIII obtained by VCCM method is transformed to this coordinate system by the following equations.

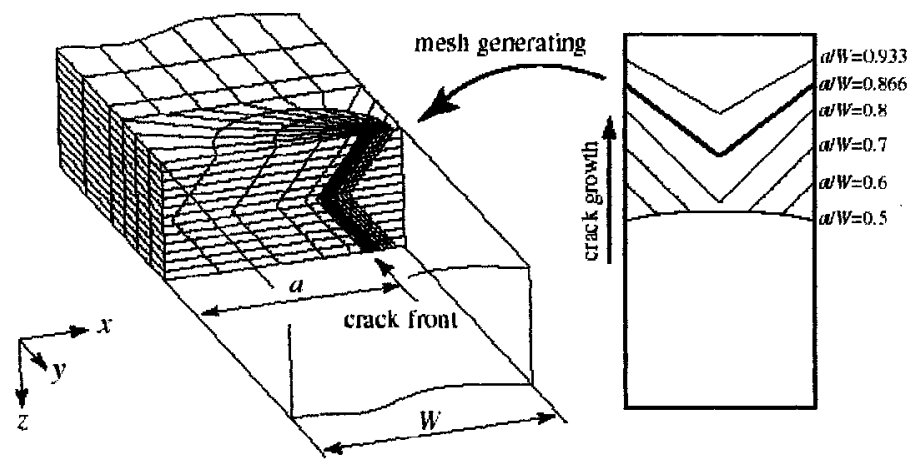

Figure 9 FEM model of growing crack..

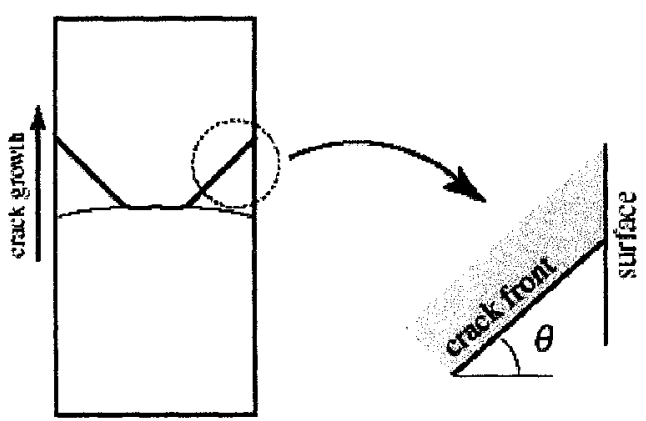

Figure 10 Crack front coordinate. 


$$
\begin{aligned}
& G_{I I}^{\prime}=G_{I I} \cos \theta-G_{I I I} \sin \theta \\
& G_{I I I}^{\prime}=G_{I I} \sin \theta+G_{I I} \cos \theta
\end{aligned}
$$

where $\theta$ is defined in Figure 10. Using these new GII and GIII values, the stress intensity factors KII and KIII are evaluated.

The changes of the distributions of stress intensity factors along the crack front are shown in Figure 11(a) and (b). Figure 11(a) shows the change of the KII distribution with the fatigue crack growth, and Figure 11(b) is that of KIII. Mode I component, $\mathrm{KI}$, is nearly zero for every cases, and are not shown here. The important point in these figures is the location of the peak values of KII and KIII for each a/W value. Before the mixed mode fatigue crack growth, $a / \mathrm{W}=0.5$, the peaks of $\mathrm{KII}$ and $\mathrm{KIII}$ are at both specimen surfaces. The initial fatigue crack growth occurs at specimen surfaces at first. As the fatigue crack grows, the peak points of both figures move to inside of the specimen. It is noticed that the peak points are the intersecting points of fatigue crack with the initial crack front. After two cracks meet at the mid-plane, the peak points of KIII are the mid-plane of the specimen in the following crack growth. At the mid-plane, KII values keep to be zero because the crack front configuration is symmetric with respect to the mid-plane. It is estimated that the fatigue crack growth occurs mainly at the peak points of stress intensity factors, and other crack front area follows to the peak points.

The fatigue crack growth direction is estimated. By Erdogan-Sih criterion, the fatigue crack grows to the direction where the crack opening stress becomes the maximum. In the mode I+II mixed mode condition, it is widely known that this criterion predicts the crack growth direction very well. In this study, the maximum crack opening stress direction is obtained including the mode III loading. Another criterion to predict the fatigue crack growth is also used. As the fatigue crack growth occurs mainly by shear loading, the maximum shear stress criterion is employed. The directions of the maximum crack opening stress and the maximum shear stress are evaluated[6,7]. The distributions of these directions along the crack front are shown in Figure 12 (a) and (b). They are the results for $\mathrm{a} / \mathrm{W}=0.5$ and 0.8 , respectively. The predicted crack growth direction at the specimen surface for $a / W=0.5$ is about 30 degree from the initial crack direction by Erdogan-Sih criterion. It becomes about 60 degree for $a / W=0.8$. As shown in Figure 8, the initial fatigue crack grows along 0 degree, and it does not agree with these results. For other cases considering the fatigue crack growth, similar results are obtained by using Erdogan-Sih criterion, and they don't agree with the real crack growth direction. On the other hand, the direction predicted by the maximum shear stress criterion keeps nearly zero along the crack front. It does not change by the change of the $\mathrm{a} / \mathrm{W}$ value. By the experimental observation, it is obvious that this criterion predicts the fatigue crack growth direction very well.

\section{EVALUATIOIN OF THE CRACK GROWTH RATE}

The crack growth rate is evaluated with the change of the stress intensity factor range. In the I+II mixed mode condition, an equivalent stress intensity factor is proposed by combining KI and KII. In this study, the equivalent stress intensity factor is calculated by the following equation.

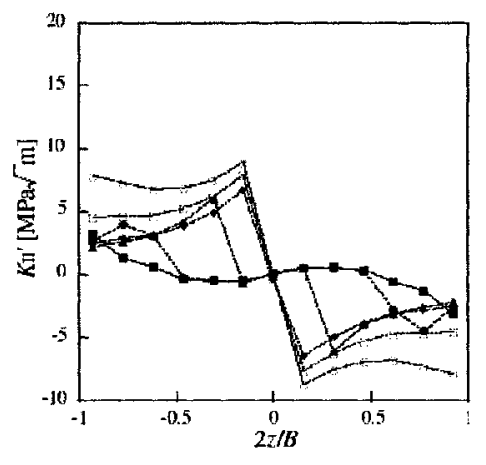

(a) KII distribution.

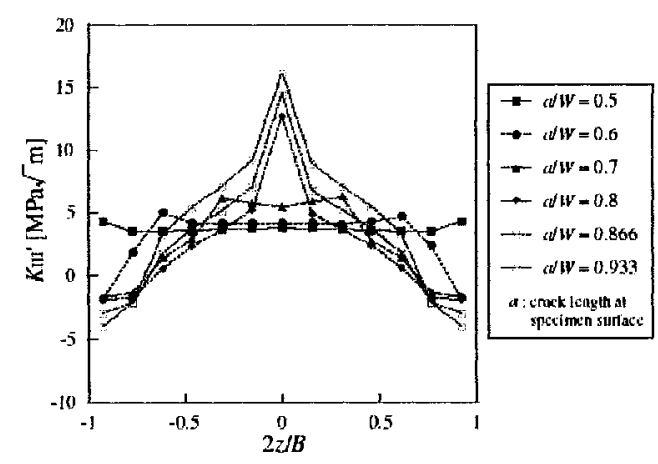

(b) KIII distribution.

Figure 11 Changes of KII and KIII distributions due to the fatigue crack growth. 


$$
K=\sqrt{\left(1-v^{2}\right) K_{I I}^{2}+(1+v) K_{I I I}^{2}}
$$

where KII and KIII are evaluated using GII and GIII values calculated by equations (1) and (2). The equivalent stress intensity factor range is obtained along the crack front. The change of the distribution of this value due to the fatigue crack growth is shown in Figure 13. It is noticed that the maximum stress intensity factor range value moves by the fatigue crack growth. Before both fatigue cracks from specimen surfaces meet at the mid-plane, the maximum point appears at the intersection of the fatigue crack front with the initial crack front. After meeting, the maximum point is at the mid-plane of the specimen. It is estimated that the fatigue crack growth rate is controlled by the maximum value of the stress intensity factor range. Then the relation between $\mathrm{da} / \mathrm{dN}$ and the maximum stress intensity factor range is plotted in Figure 14. In this figure, the results of pure mode I fatigue test are also plotted. It is shown that the fatigue crack growth rate of mixed mode is a little bit smaller than that of mode I. But the threshold values of both results are similar to each other.

\section{CONCLUDING REMARKS.}

The mode II + III mixed mode fatigue test is conducted using a single-axis testing machine. By giving the static mode I loading, the contact between fatigue crack surfaces could be avoided. It is found that the fatigue crack growth occurs at the surface of the specimen at first, and then grows to inside. It is estimated by the changes of the distributions of stress intensity factors along the crack front. By defining an equivalent stress intensity factor range, it is found that the mixed mode fatigue crack growth rate is nearly same with that of pure mode I fatigue.

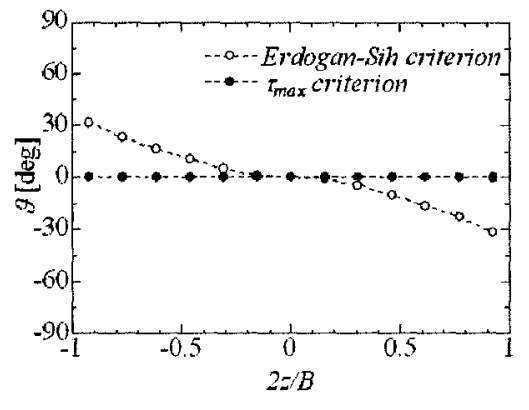

(a) $\mathrm{a} / \mathrm{W}=0.5$

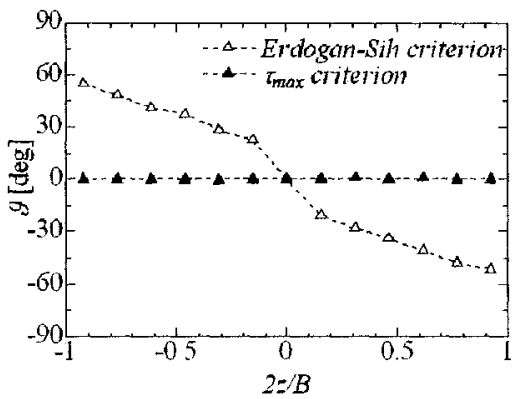

(b) $\mathrm{a} / \mathrm{W}=0.8$

Figure 12

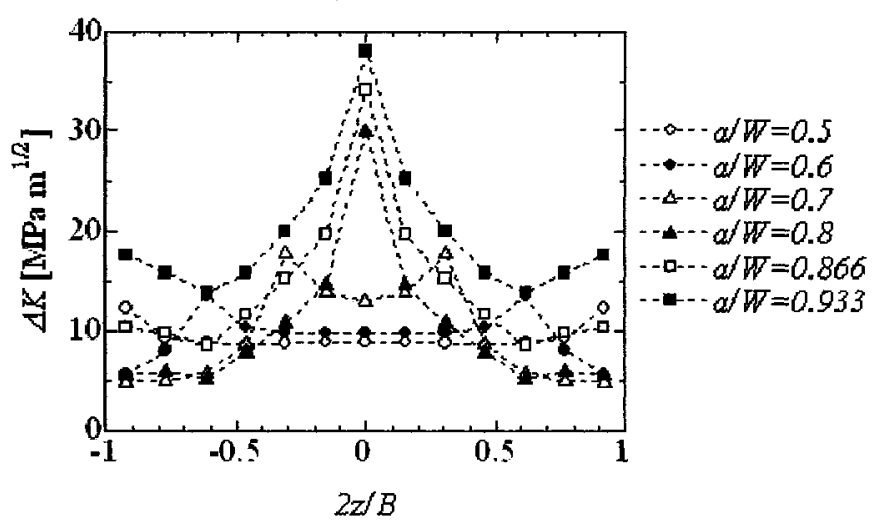

Figure 13 Distributions of equivalent stress intensity factor range. 


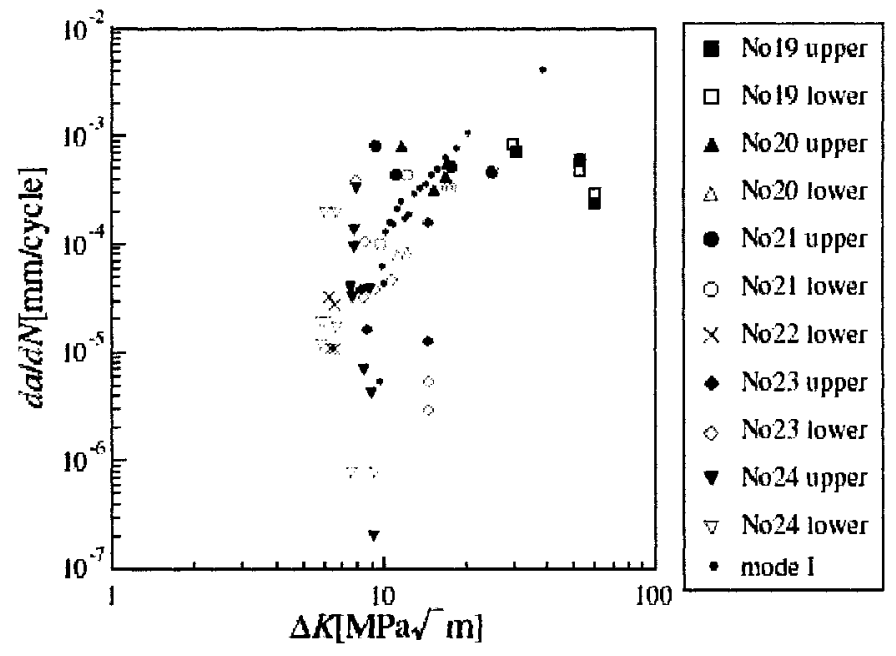

Figure 14 Fatigue crack growth rate.

\section{ACKNOWLEDGEMENT}

This work was conducted under Research Committee on System Integrity of Rotational Machinery by Tokyo Electric Power Co.

\section{REFERENCES}

1. Richie, R.O.,F.A.McClintock, H.Nayeb-Hashemi, and M.A.Ritter, Metallurgical Transaction A, vol. 13A, 1982, p.101-110.

2. Erdogan, F., and Sih, G.C., J. Basic Engineering, Trans., ASME, Ser.D, 1963, p.519-527.

3. S.K.Maiti, Engineering Fracture Mechanics, vol.41, No.3, 1992, p. 339-342.

4. A.Otuka, K.Mori and T.Miyata, Engineering Fracture Mechanics, vol.7 1975 , p.429.

5. W.A.Spitzig, ASTM STP vol.90 1969 , p. 453.

6. Iida, S. and Kobayasi, A.S. Trans. ASME, Ser.D, 1969, p.764.

7. Chen, X.A., Engineering Fracture Mechanics, vol. 24 1986, p. 127. 